\title{
DEVELOPMENT METHODOLOGY FOR OPTOMECHATRONIC SYSTEMS USING THE EXAMPLE OF A HIGH-RESOLUTION PROJECTION MODULE
}

\author{
Ley, Peer-Phillip; Knöchelmann, Marvin; Kloppenburg, Gerolf; Lachmayer, Roland \\ Institute of Product Development
}

\begin{abstract}
In the last few years there has been a noticeable change in the development of headlamp systems in the field of vehicle lighting technology. Starting with adaptive front-lighting systems via Matrix LED systems, high-resolution headlamps will provide more safety in road traffic in the near future.

For the implementation of high-resolution headlamps various spatial light modulators and light generating technologies can be applied. The emitted light of the light source is directed via an illumination optics onto the modulator and a projection optics is applied to image the spatial light modulator into the traffic area. The formerly mechatronic systems are thus increasingly become optomechatronic systems. Therefore, the optic design must be taken into account in the early development phase of these systems.

In this paper we present a methodical approach to describe the optic design for optomechatronic systems. This approach can be used to develop efficient and high-intensity optomechatronic systems using various spatial light modulators and light generating technologies. Conclusively we demonstrate an exemplary application of the methodology on a high-resolution projection module.
\end{abstract}

Keywords: Optimisation, Mechatronics, Product modelling / models

Contact:

Ley, Peer-Phillip

Institute of Product Development

Optomechatronic

Germany

ley@ipeg.uni-hannover.de

Cite this article: Ley, P.-P., Knöchelmann, M., Kloppenburg, G., Lachmayer, R. (2019) 'Development Methodology for Optomechatronic Systems Using the Example of a High-Resolution Projection Module', in Proceedings of the 22nd International Conference on Engineering Design (ICED19), Delft, The Netherlands, 5-8 August 2019. DOI:10.1017/ dsi.2019.261 


\section{INTRODUCTION}

The development of efficient LED systems has led to a change in many areas of lighting and lighting technology. Especially in the vehicle lighting technology where conventional headlamps, consisting of a discharge lamp and a reflector or lens, are increasingly being replaced by systems consisting of LED arrays and complex optical systems. With a correspondingly high amount of LEDs and their individual control within the array, an increased functional integration can be implemented. In addition to that, the chosen optical concept to implement the system, offers great freedom in the area of vehicle design.

A further step in this development is the introduction of high-resolution headlamps, which allow the generation of individually adapted light distributions in order to achieve an optimal illumination of the traffic area. The light distribution of the headlamps consists of a variety of pixels, similar to projectors in the entertainment industry. Depending on the technology used for the image generation and implementation of the optical system, a various amount of pixels can be realized, which results in a range of different functions. While a small amount of pixels allows the individual illumination of the traffic area without dazzling other road users, more pixels allow the projection of information onto the road.

In lighting devices, in particular in the field of vehicle lighting technology, the optical system gains in importance. Consequently a change from manageable mechatronic systems to elaborate optomechatronic systems occurs. In order to meet the new challenges in the development of these systems, more than ever the application of methodical tools is required. The V-Model from the VDI 2206 (2004), which is an established method for the development of mechatronic systems, can only be applied to a limited extent in the development of elaborate optomechatronic systems. The increasing importance of the optical system must be considered as an optic design in the early phase of the system design. Therefore, it is necessary to develop a new methodical approach, which describes the optic design within the V-model.

\section{SYSTEM STRUCTURE AND LIGHT PATH OF HIGH-RESOLUTION HEADLAMPS}

First a comparison between an AFS headlamp and a high-resolution headlamp will be made, to derive the challenges and innovations in the development of high-resolution headlamps. Figure 1 shows the system structure of a high-resolution headlamp. To record information from the environment, such as number and position of other road users, lane position, traffic signs, etc., different sensors (front camera, lidar and radar sensors) are used. Including further information from radio-based Car2X functions, all information is collected in a centralized information processing unit. The results are control commands to generate suitable projections onto the road in the respective driving situation.

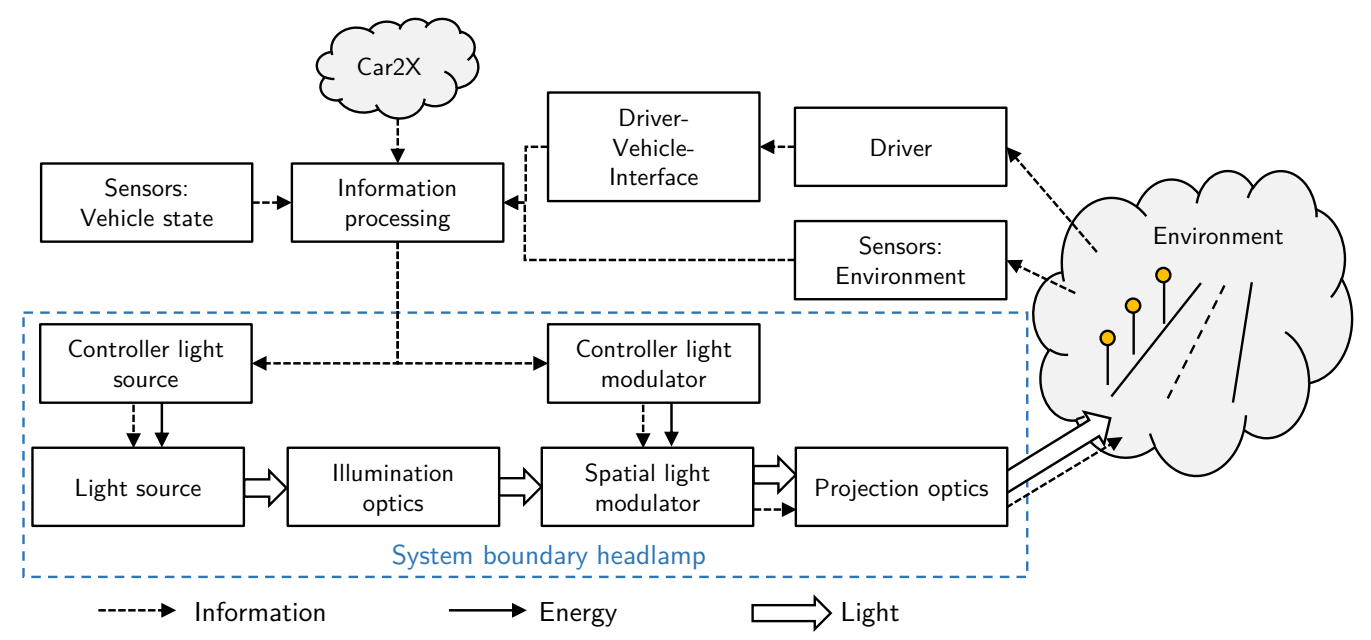

Figure 1. System structure of a high-resolution headlamp (Knochelmann et al. (2019))

To a limited extent sensors are also used in AFS headlamps in order to generate the current suitable light distribution ECE123 (2010) depending on vehicle speed and steering angle. The required data are evaluated by the control unit for the light, which can be located directly in the headlamp or outside. In both systems, the driver also receives information from the environment and converts it into driving maneuvers. The driver can, for example, control assistance functions via the driver-vehicle interface. 
In AF-Systems, different light functions are realized e.g. by a special roller. Depending on the desired light function, the roller is rotated so that it acts as an adaptive aperture within the beam path. The projection optics images the contour of the roller into the road space. The light path (light source, aperture, projection optics) is rotatably mounted and can be pivoted in both the vertical and horizontal axis, so that a dynamic cornering light can be realized. Xenon discharge lamps are predominantly used as light sources (Lachmayer (2016)).

High-resolution headlamps use a completely different approach to create and model visible light in automotive lighting technology. The light path of a high-resolution headlamp comprises the light source, illumination optics, the spatial light modulator and the projection optics. It can be subdivided into an illumination path and projection path (Figure 2). To model the light distribution including the desired information different technologies from video and entertainment technology (DMD, LCD) can be used. Kloppenburg et al. (2016) give an overview of the technologies and their properties. Digital micromirror devices (DMDs) for instance consist of a few hundred thousand switchable micromirrors. Depending on the position of the micromirros the emitted light from the light source is being reflected onto an absorber or into the projection optics, which subsequently projects the modeled image onto the road. The individual mirrors thus become pixels of a high-resolution image. Since it is possible to implement a digital cornering light with the use of this technology, moving parts such as the actuator and rotatable suspension of the entire optical path for the cornering light are being dropped (Pfullmann et al. (2017)).

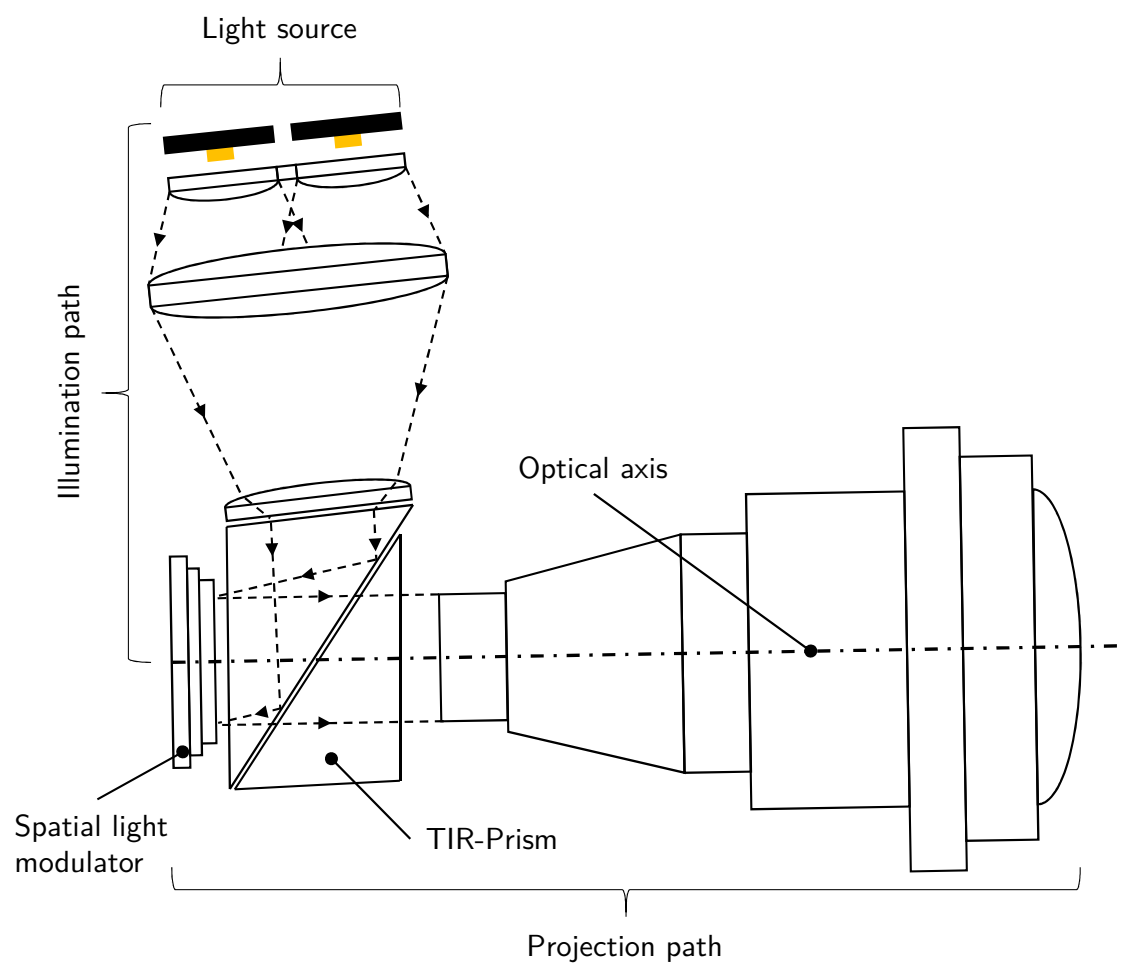

Figure 2. Light path of a high-resolution headlamp (Ley et al. (2019))

The comparison of these two systems shows that the interconnection of the individual components and the number of used data from the environment and vehicle increases. In the system structure of the high-resolution headlamp (Figure 1) a separate block for information processing is shown, which can be a central vehicle computer. Within the system boundary of the headlamp, the spatial light modulator is added as a new component, which requires a precise adjustment and suitable control. LEDs are almost exclusively used for light generation due to their high efficiency and durability. High-resolution headlamps exhibit significant differences to previous headlamps, resulting particularly in the following challenges in the development of these systems:

- The increasing interconnection using the vehicle camera and other sensors

- Required data and interfaces must be defined in early stages of the development process and require frequent exchange of domain-specific development teams or in interdisciplinary teams. 
- The application of spatial light modulators to generate the high resolution

- Spatial light modulators are used for the first time in headlamps and partly in general for the first time in automotive engineering.

- The increasing importance of the optical system

- The elaborate optical system of a high-resolution headlamp comprises of an illumination optics and projection optics which require a precise adjustment within the light path to set up a highly efficient system.

- The increased application of semiconductor light sources

- Solid-State light sources such as LEDs and laser diodes are supplanting and replacing conventional light sources like xenon discharge lamps. They differ significantly in terms of control, operating behavior and the required cooling (Kusch et al. (2017)).

High-resolution headlamps exemplify future challenges in the development of highly interconnected and highly efficient optomechatronic systems using new technologies. The methodical development of such systems requires an adaptation of existing methodologies to be able to deal with these increasing challenges.

\section{Challenges in DEVELOPING EFFICIENT High-RESOLUTION HEADLAMPS}

For mechatronic systems, the V-model from VDI 2206 (2004) is an established method and will also be applied here in a modified version (Figure 3). In the V-Model it is recommended to generate models at various levels of abstraction for the system design. A topological model shows the relations of the components to each other. At the next level, essential physical relations are described. Subsequently, another abstraction should lead to a mathematical model in order to be able to record the properties of different domains in a model and to work out cross-domain solution concepts. After the cross-domain system design, a specific design within the individual domains follows in the V-Model.

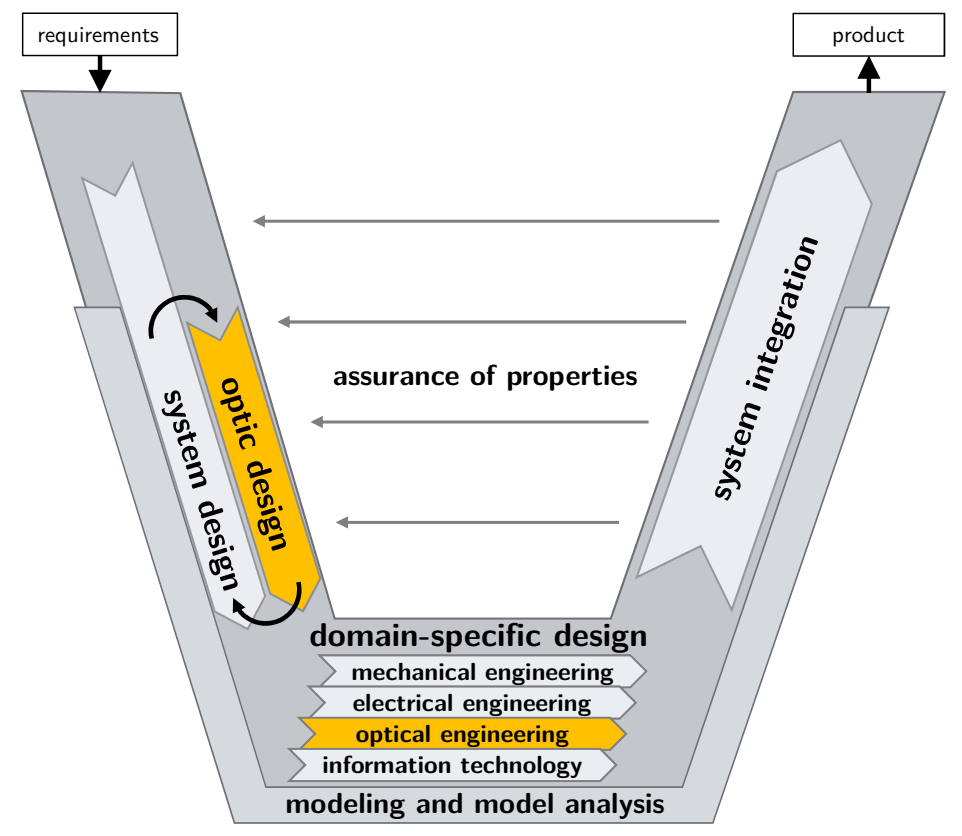

Figure 3. V-model based on VDI 2206 (2004) with supplementary optic design

The comparison of the AFS headlamp and the high-resolution headlamp shows that optical systems of future headlamps are significantly larger and more complex. Therefore a complete independent design of the illumination optics and projection optics is not possible. The illumination strategy, i.e. how the spatial light modulator is illuminated, and the type of distortion, i.e. how the projection optics illuminates the traffic space, are directly dependent on each other. Lighting strategy and type of distortion can be defined in the requirements, but must be checked for a high-intensity and efficient system during 
the optic design phase and adjusted if necessary. Therefore the optical system leads to a cross-domain relation. In order to detect target conflicts early on and to adapt the requirements, the optic design must already be part of the system design.

The concept of the optical system for a high-resolution headlamp results primarily from the lighting requirements which have to be met with this system. Also in the mechanical and electrical domain many requirements are defined. However, the optical system permanently influences the domain-specific design of mechanical and electrical components. For example, in most cases it is possible to implement the mechanical socket of a lens at another position to maintain the functional implementation of the optical system. Conversely, it is not possible to reposition a lens within the optical system so that the mechanical socket can be positioned at the planned location. Electrical components which serve for information processing in order to generate control commands for an adapted light distribution in the respective driving situation are essential for high-resolution systems. These can nevertheless be arranged directly in the headlamp or outside to fulfill the function. However, the spatial light modulator as an optical element within the optical system requires precise alignment and positioning dependent on the illumination optics and projection optics.

The above mentioned direct dependencies between the individual optical components and the crossdomain relationship between mechanical and electronic components requires an adapted approach for the methodical development of high-resolution headlamps based on the V-model. The specific methodical approach in the design phase of the optical system is explained in the following section 4 and then shown by an example in section 5 .

\section{METHODICAL APPROACH FOR THE OPTIC DESIGN WITHIN THE V-MODEL}

In this section a methodical approach (Figure 4) is introduced to describe the optic design based on the challenges during the development of high-resolution headlamps (Section 3) and the V-Model. The approach is described by using the SysML modeling language. Individual steps within the approach are defined by sequential and parallel processes of individual actions. The combination of actions and their relation through control flows forms a sequence that describes the development of the optical system for high-resolution headlamps. The individual activities and their meaning to model the optic design as well as their sequence are explained below.

\section{Definition of requirements}

First, the definition of photometric requirements, which are recorded in a request list, is required. The lighting requirements are based on light functions (low beam, high beam, information projection, etc.), photometric quantities (luminous flux, illuminance, luminance, etc.) and geometric sizes of the optical system (length of optical system, number of optical elements, lens diameter, etc.), which should be fulfilled by the system.

\section{Select spatial light modulator}

Within the high-resolution headlamp, the light modulating element represents the elementary and at the same time limiting component to implement the lighting requirements. The smaller the active area of the light modulator, the less light can be modulated. Consequently, after the definition of requirements, the action "Select spatial light modulator" results. Within this action the clarification of the applied modulator technology, its size of the active area and aspect ratio is required. In addition to that the determination of the Étendue of the spatial light modulator (simplified: product of the active area of the modulator and the acceptance angle of the modulator), which serves as a conserved quantity in optical systems, is necessary.

\section{Select light source}

With regard to the selection of the spatial light modulator, the choice of the applied LED light source requires the clarification of the amount of LEDs to illuminate the active area of the modulator. Deciding on a reasonable number of LEDs to implement an efficient and high-intensity system, the Étendue of the light source, thus the product of the emission area and emission angle of the LED, has to be taken into account. Subsequently, the determination of the Étendue as well as its comparison between the 
spatial light modulator and the light source is required. Assuming an ideal optical system to illuminate the active area of the modulator, an efficient system can be assumed for a comparable value of Étendue.

\section{Determine system architecture}

The system architecture deals with the spatial arrangement of the optical components within the optical system and consequently also influences the system Étendue. Using a spatial light modulator, the optical system can basically be subdivided into an illumination system - to illuminate the light modulator - and a projection system - for the projection onto the street. The spatial arrangement of illumination system and projection system is tied to the chosen modulator technology. Both optical systems serve to fulfill different functions and are nevertheless functionally as well as spatially directly dependent on each other.

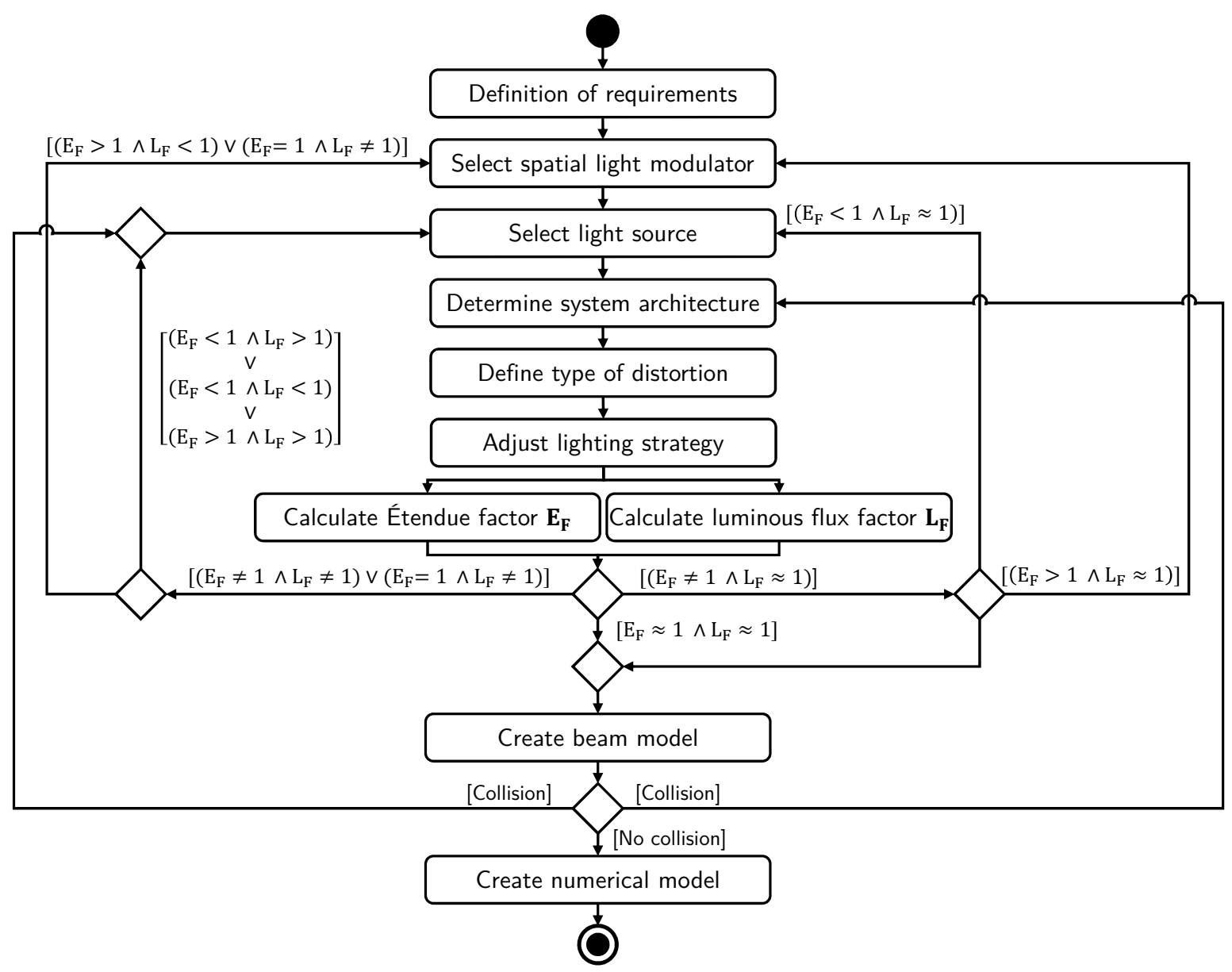

Figure 4. Methodical approach for the optic design

\section{Define type of distortion}

The light reflected by the light modulator into the projection optics can be imaged into traffic space using various optical concepts for the projection optics. In general, these concepts can be subdivided into different types of distortion (distortion: not to scale representation of an object). A radial design concept for the projection optics allows, for example, the redistribution of the illuminance and at the same time a variable pixel size in the traffic area (Wolf et al. (2016), Wolf et al. (2017)). Anamorphic concepts may require a defined illumination strategy, thus shaping the light from the light source onto the active area of the modulator. Accordingly, the type of distortion has a significant influence on the strategy to illuminate the light modulator but also the optical efficiency.

\section{Adjust lighting strategy}

The lighting strategy deals with the way the light modulator is illuminated. In principle, a distinction can be made between homogeneous and inhomogeneous illumination of the active modulator area (Ley et al. 
(2018)). Homogeneous illumination allows a maximum system Étendue, which means that a highly efficient system can be implemented. In case of an inhomogeneous illumination, the full potential of the system can not be exploited. As a result the maximum possible luminous flux on the modulator surface is lower than in case of an homogeneous illumination. However, a suitable inhomogeneous illumination of the modulator surface can reduce the distortion of the projection optics. Therefore an illumination strategy adapted to the type of distortion can increase the efficiency of the projection optics, which ultimately increases the overall system efficiency (Knöchelmann et al. (2018)). Following the adjustment of the lighting strategy, the calculation of a so-called Étendue factor is required to implement an efficient system.

\section{Calculate Étendue factor}

The Étendue factor $E_{F}$ can be understood as the ratio of the Étendue of the light source $\mathrm{G}_{\mathrm{LQ}}$ to the Étendue of the spatial light modulator $\mathrm{G}_{\mathrm{LM}}$ and serves as an indicator to evaluate the system efficiency. A high efficiency system is characterized by an Étendue factor of about one $\left(\mathrm{E}_{\mathrm{F}} \approx 1\right)$. If the Étendue factor is significantly larger or smaller than one, the system is distinguished by inefficiency. However, an inefficient system is not equivalent to the failure of fulfilling the defined lighting requirements. The rough determination for the fulfillment of the lighting requirements is taken into account by the determination of a so-called luminous flux factor.

\section{Calculate luminous flux factor}

The luminous flux factor $\mathrm{L}_{\mathrm{F}}$ can be understood as the ratio of actual luminous flux to the target luminous flux, on the active area of the modulator and serves to verify the feasibility of the required lighting functions. The target luminous flux is the luminous flux on the active area of the modulator, which is required to implement the light functions. The actual luminous flux accordingly represents the luminous flux on the active area of the modulator which is effectively directed from the light source to the modulator using the illumination optics. Similar to the Étendue factor three cases can occur. A luminous flux factor of approximately one and greater than one $\left(\mathrm{L}_{\mathrm{F}} \approx 1, \mathrm{~L}_{\mathrm{F}}>1\right)$ indicates a system in which the required lighting functions can be implemented. If the luminous flux factor is less than one $\left(\mathrm{L}_{\mathrm{F}}<1\right)$, the required lighting functions can not be implemented. Finally a case analysis has to be made, based on the calculated Étendue and luminous flux factor.

\section{Case analysis}

In general, the cases that can occur can be classified into three categories. Category 1 $\left[\left(\mathrm{E}_{\mathrm{F}} \neq 1 \wedge \mathrm{L}_{\mathrm{F}} \neq 1\right) \vee\left(\mathrm{E}_{\mathrm{F}}=1 \wedge \mathrm{L}_{\mathrm{F}} \neq 1\right)\right]$ defines cases where the required lighting functions can not be implemented due to a too low luminous flux factor. For these cases, the methodical approach provides loops to adjust either the light source or the spatial light modulator. Category 2 $\left[\mathrm{E}_{\mathrm{F}} \neq 1 \wedge \mathrm{L}_{\mathrm{F}} \approx 1\right]$ discribes cases in which systems with the required light functions can be implemented, but the modeled system is characterized by inefficiency due to an Étendue factor distinctly unequal to one. If these cases occur, two decisions can be made within the approach. Either the light source or the light modulator is adapted via a loop or the implementation of a beam model can be started, since an implementation of the lighting requirements can be realized. Category $3\left[\mathrm{E}_{\mathrm{F}} \approx 1 \wedge \mathrm{L}_{\mathrm{F}} \approx 1\right.$ ] describes the case that a system of highest efficiency can be implemented while fulfilling the required lighting functions, which allows to develop the beam model right away.

\section{Create beam model}

The beam model serves as the basis to describe the behavior of the beam path. Within the optical system, the beam path is described using paraxial optics, which is a simplification of geometric optics. Critical system configurations, which for example lead to the collision of optical elements with each other or the illumination path or projection path, can be identified and eliminated through loops within the methodical approach. If there is no collision (anymore), the development of a numerical model can be started.

\section{Create numerical model}

In the final step of the methodical approach, a numerical model of the optical system will be developed using computer-aided optical simulation techniques. The optical system is modeled from the light 
source to the image plane (road). The modeling depth has a decisive influence on the significance of how well the system fulfills the lighting requirements in reality. Therefore, it is necessary to consider influences of aberrations, scattered radiation and reflexion radiation as well as alignment errors. If the defined lighting requirements are insufficient, it is possible to go through optimization loops. The optimization loops are based in part on empirical values and the gradual adaptation of decisions within the methodical approach.

\section{EXEMPLARY APPLICATION OF THE METHODICAL APPROACH}

For the exemplary application of the methodical appraoch, a high-resolution projection module for a vehicle headlamp is designed. The goal is to implement an efficient and high-intensity projection module, in which the technology for image and light generation can be chosen freely. The lighting requirements for the projection module are listed in Table 1. Lee et al. (2011) show, that a suitable distance to project symbols is at around 12,6 meters. Therefore, a projection distance of $5-15$ meters for the projection module is chosen.

Table 1. Requirements for the high-resolution projection module

\begin{tabular}{|l|c|} 
Requirements & Value \\
Projection distance & $5-15 \mathrm{~m}$ \\
Opening angle (horizontally) & $11,42^{\circ}$ \\
Opening angle (vertically) & $6,77^{\circ}$ \\
Luminous flux & $1500 \mathrm{~lm}$ \\
Illuminance (Hotspot) & $150 \mathrm{~lx}$
\end{tabular}

The used technology of the light modulating element for the high-resolution projection module can be LCD, DMD or Liquid Crystal on Silicon (LCoS). LED arrays, which ultimately also modulate light are not suitable as a light modulator due to the small amount of pixels. The operating principle of LCD and LCoS requires the use of polarized light. When implementing an efficient system this fact leads to a higher effort in the design of the optical system. Therefore the application of the DMD technology is preferred to implement the projection module using an unpolarized white light source (Held et al. (2018)). The applied DMD (Table 2) (Texas Instruments (2018)) is certified for automotive applications and the micromirrors exhibit a tilt angle of $\theta_{\text {tilt }}=12^{\circ}$. Due to the high luminous efficacy and durability compared to other light sources, LEDs are preferred to illuminate the DMD. The Étendue of the light source is directly dependent on the angle of emission respectively the acceptance angle of the following optics. Thus, the subsequent optics are of particular importance in terms of system efficiency. At the same time, the acceptance angle defines the Étendue of the light source (Table 3). For a system of maximum efficiency, an equivalent Étendue value of the LED and DMD is required.

Table 2. Specifications of the DLP5530-Q1

\begin{tabular}{|l|l|l|} 
Feature & Symbol & Value \\
Active area & $A_{S L M}$ & $77.50 \mathrm{~mm}^{2}$ \\
Aspect ratio & $-/-$ & $2: 1$ \\
Tilt angle & $\theta_{\text {tilt }}$ & $12^{\circ}$ \\
Étendue & $G_{S L M}$ & $10.52 \mathrm{~mm}^{2} \mathrm{sr}$
\end{tabular}

Table 3. Specifications of the LED

\begin{tabular}{|l|l|l|} 
Feature & Symbol & Value \\
Emission surface & $A_{L E D}$ & $4.11 \mathrm{~mm}^{2}$ \\
Aspect ratio & $-/-$ & $2: 1$ \\
Emission angle & $\theta_{E m i}$ & $\pm 90^{\circ}$ \\
Étendue @ $\pm 65^{\circ}$ & $G_{L E D}$ & $10.606 \mathrm{~mm}^{2} \mathrm{sr}$
\end{tabular} \mid

The system architecture, which discusses the spatial arrangement of the optical systems (illumination system and projection system), can be differentiated into a telecentric and non-telecentric architecture when using a DMD as a spatial light modulator (Ley et al. (2019)). Regardless of the chosen architecture, the DMD technology requires an illumination of the active area of the modulator at an angle $\theta_{I l l}=12^{\circ}$. In this example a non-telecentric system architecture for the high-resolution projection module is chosen. Using this system architecture, the DMD requires an additional tilt of $12^{\circ}$ to separate the illumination path from the projection path, resulting in $\theta_{I l l}$ at $24^{\circ}$. The Étendue of the light modulator can be determined for the respective system architecture using the following equation.

$$
G_{S L M}=A_{S L M} \cdot \sin ^{2}\left(\theta_{t i l t}\right) \cdot \cos \left(2 \cdot \theta_{I l l}\right)
$$


The initial Étendue of the DMD therefore changes to $7.04 \mathrm{~mm}^{2} \mathrm{sr}$. In order to design an efficient system, it is necessary to adjust the acceptance angle of the optics from $\pm 65^{\circ}$ to $\pm 48^{\circ}$ so that the Étendue of the light source changes to $7.13 \mathrm{~mm}^{2}$ sr. The lighting requirements demand an illuminance of $150 \mathrm{~lx}$ in the hotspot which can be implemented by using a radial distortion for the projection optics. For this distortion concept, a homogeneous illumination of the DMD is suitable to use the maximum Étendue. By creating a beam model, critical system configurations can be identified and eliminated by looping within the methodical approach before in the final step the numerical model (Figure 5) is set up. in this step the optical system is designed from the light source to the image plane by means of computer-assisted optical simulation techniques.

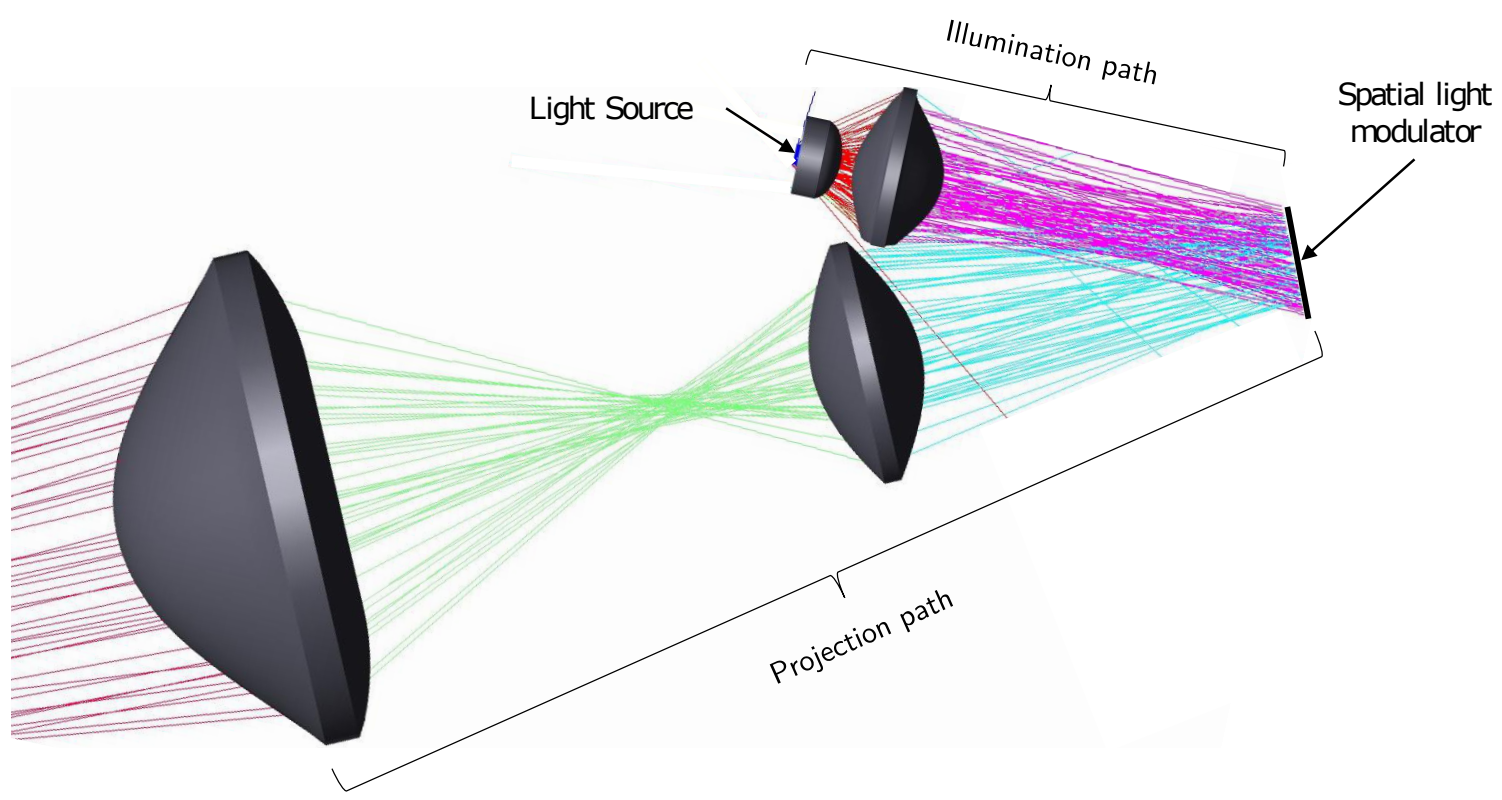

Figure 5. Numerical model of the high-resolution projection module

In first approximation each optical element within the optical system is pre-defined by a sequence of surfaces. Using merit functions different target values and constraints of variables, e.g. lens thickness, lens diameter or the distances between the individual optical elements, can be defined. This first approximation allows fast ray tracing, optimization and tolerancing using geometric optics. Once the defined target values and constraints have been met, the model, which has been set up in a first approximation, is converted into a realistic model (Figure 5) and analyzed with regard to aberrations, scattered and reflected radiation as well as alignment errors. Optimization loops can be made on the basis of empirical values, by adapting the numerical model and stepwise adapting decisions within the methodical approach until the numerical model fulfills the requirements.

\section{CONCLUSION}

In this paper we presented a methodical approach based on the V-Model from the VDI 2206 (2004) to describe the optic design for optomechatronic systems like high-resolution headlamps. The applied modeling language to describe the optic design is SysML. Starting with the photometric requirements for the system, the required steps to implement the optical system are then sequentially requested. First the applied technology for the spatial light modulator has to be chosen. Simultaneously the size of the active area and the aspect ratio of the spatial light modulator has to be defined. Next, a suitable light source to illuminate the modulator has to be chosen. The choice is based on the Étendue of the modulator. Subsequently, the system architecture of the optical system, which depends on the modulator technology must be determined. The definition of the distortion of the projection optics and the strategy to illuminate the light modulator are directly dependent on each other. Therefore an illumination strategy adapted to the type of distortion is required to set up an efficient and high-intensity system. With the following case analysis based on the determination of an Étendue factor and luminous flux factor, the system efficiency and the feasibility of the photometric requirements are controlled. Subsequently, the behavior of the optical system must be modeled precisely with models to verify the technical feasibility of the system. The basis for the behavioral description is the creation of a beam path model using paraxial optics, which 
is a simplification of geometric optics. The collision of optical elements with each other or between the illumination path or projection path are eliminated by a final query. The methodical approach ends with the set up of a numerical model of the optical system. In this step, the system is modeled from the light source to the image plane (road) by means of computer-assisted methods for optical simulation. Afterwards it is possible to go through optimization loops, which are partly based on empirical values and the gradual adaptation of decisions within the methodical approach.

We demonstrated the approach on an example for a high-resolution projection module for a vehicle headlamp. But in principle, this approach can be applied to any high-resolution system using spatial light modulators. It offers a structured way to design efficient and high-intensity optical systems for highresolution systems using various spatial light modulators and light generating technologies. Therefore it can be applied in the general field of video and lighting technology but also in special areas such as medical technology (Ilango et al. (2018)).

\section{REFERENCES}

VDI 2206 (2004). VDI-Richtlinie 2206 Design methodology for mechatronic systems. Berlin : Beuth Verlag.

Knöchelmann, M., Kloppenburg, G., Mozgova, I., Lachmayer, R. (2019). Methodische Entwicklung eines opto-mechatronischen Systems am Beispiel eines hochadaptiven Fahrzeugscheinwerfers. in VDI Berichte, Mechatronik 2019, Paderborn, 27-28 March, ISBN 978-3-945437-05-6.

ECE 123. (2010), Regelung Nr. 123 der Wirtschaftskommission für Europa der Vereinten Nationen (UN/ECE) Einheitliche Bedingungen für die Genehmigung von adaptiven Frontbeleuchtungssystemen (AFS) für Kraftfahrzeuge

Lachmayer, R. (2016). Beleuchtung. in Vieweg-Handbuch Kraftfahrzeugtechnik, Springer Vieweg, Wiesbaden, pp 890-906.

Kloppenburg, G., Wolf, A., Lachmayer, R. (2016). High-resolution vehicle headlamps: technologies and scanning prototype. in Advanced Optical Technologies, Vol. 5 No. 2, pp 147-155, http://doi.org/10.1515/aot-2016-0001.

Pfullmann, N., Thiel, A., Thamm, M., Plöger, R., Kloppenburg, G., Wolf, A., Lachmayer, R. (2017). From mechanical ADB systems to high resolution headlamps - new opportunities of novel headlight systems. in ISAL 2017 Proceedings, 12th International Symposium on Automotive Lighting, Darmstadt, 25-27 September.

Ley, P.-P., Lachmayer, R. (2019). Imaging and non-imaging illumination of DLP for high resolution headlamps. in Proceedings of SPIE 10932, SPIE Photonics West, San Fransisco, February 1st - 7th, http://doi.org/10.1117/12.2507436

Kusch, A., Wurz, M., Held, M. P., Wolf, A., Lachmayer, R. (2017). LED packaging with optimized heat dissipation for a micro LED array. in International Conference and Exhibition on the Integration of Materials, Devices and Systems, Smart Systems Integration, Ireland, 8-9 March.

A. Wolf, G. Kloppenburg, R. Danov, R. Lachmayer (2016). DMD Based Automotive Lighting Unit. in DGaO Proceedings, Deutsche Gesallschaft für angewandte Optik, Hannover, 17-21 May, http://doi.org/10.15488/4004

A. Wolf, G. Kloppenburg, R. Lachmayer (2017). Lighting Device, Lighting Method and Computer Program. Patent No. WO/2017/148764. Germany. [2017, September 08].

Ley, P.-P., Held, M. P., Wolf, A., Lachmayer, R. (2018). Konzepte zur Beleuchtung von Lichtmodulatoren. in DGaO Proceedings, Deutsche Gesallschaft für angewandte Optik, Aalen, 22-26 May, http://doi.org/10.15488/4085

Knöchelmann, M., Wolf, A., Kloppenburg, G., Lachmayer, R. (2018). Aktiver Scheinwerfer mit DMD-Technologie zur Erzeugung vollständiger Lichtverteilungen. in VDI Berichte 2323, Optische Technologien in der Fahrzeuglichttechnik, Karlsruhe, 5-6 June.

Lee, T. and Park, G. and Kim, H. and Park, I. and Lee, K., Ahn, B. (2011). The Development of the Automotive Headlamp using the DMD. in ISAL 2011 Proceedings, 9th International Symposium on Automotive Lighting, Darmstadt, 26-28 September.

Held, M. P., Ley, P. P., Wolf, A., Lachmayer, R. (2018). Anforderungen an die Ausleuchtung von Lichtmodulatoren. in DGaO Proceedings, Deutsche Gesallschaft für angewandte Optik, Aalen, 22-26 May, http://doi.org/10.15488/4084

Texas Instruments (2018). DLP5530-Q1 0.55-inch 1.3 Megapixel DMD for Automotive Interior Display. http://www.ti.com/lit/ds/symlink/dlp5530-q1.pdf

Ilango, S., Knöchelmann, M., Kahrs, L. A., Wolf, A., Ortmaier, T., Lachmayer, R. (2018). User evaluation study on illumination requirements to design an augmented reality projector for open liver surgery. in Proceedings of SPIE 10693, SPIE Optical Systems Design, Frankfurt, 14th - 17th May, http://doi.org/10.1117/12.2309667 